\title{
SUR LES COUCHES DE NIVEAU ÉLECTROMAGNÉTIQUES
}

PAR

\author{
E. BELTRA M I
}

a PAVIE.

Tout le monde connait les élégantes propriétés des surfaces et des couches de niveau par rapport aux masses agissant d'après la loi de Newton, ainsi que la belle application que ces propriétés reçoivent dans l'étude des systèmes ellipsoïdaux.

Des propriétés tout-à-fait analogues ont lieu par rapport aux actions électromagnétiques. Dès 1872 , dans une courte note insérée au Nuovo Cimento, j’ai indiqué des systèmes de courants, auxquels j’ai donné le nom (qui peut-ètre n'a pas été parfaitement bien choisi) de solenoïdes neutres, et qui présentent des analogies très-frappantes avec les couches de niveau. J'ai montré, un peu plus tard (en 1874), dans des Recherches sur la cinématique des fluides, que l'on rencontre une application très-simple de ces systèmes chez les surfaces de second ordre: seulement ces surfaces ne sont pas, cette fois, des ellipsoïdes, mais des hyperboloïdes à une nappe.

J'ai quelque raison de croire que la connaissance de ces propriétés n'est pas aussi généralement répandue qu'elle pourrait l'être. C'est pourquoi je prends la permission d'en présenter un exposé succinct aux lecteurs de ce Journal, d'autant plus que des recherches postérieures me permettent d'en abréger considérablement la démonstration. Je m'étais d'abord servi $\mathrm{du}$ théorème de Green, et ce procédé est sans doute le plus naturel et le plus direct, lorsque l'on tient compte d'une remarque due à $\mathbf{M}$. W. 'Тномson (Reprint, §5 55). Mais les fonctions qu'il faut considérer n'étant 
pas, en général, monodromes, l'emploi de ce théorème exige, comme on sait d'après M. Helmhostz, quelques modifications qui compliquent un peu les raisonnements. Je suivrai donc une autre voie, en maippuyant sur les formules qui servent à définir les systèmes de courants dont l'action extérieure est la mème que celle d'un corps magnétique donné. J'ai eu tout récemment l'occasion d'établir ces formules d'une manière rigoureuse, dans une Note communiquée à l'Institut Royal de Milan (séance du I 2 Avril I 883).

Je vais d'abord rappeler ees formules, ainsi que les conventions sur lesquelles elles reposent.

Soit $S$ l'espace occupé. par un corps magnétique et soient $\alpha, \beta, \gamma$ trois fonctions monodromes, continues et finies des coordonnées $x, y, z$, représentant les composantes suivant les axes du inoment magnétique (rapporté à l'unité de volume) au point $(x, y, z)$ de l'espace $S$. L'action que le corps exerce sur un point extérieur quelconque est identique, comme on sait d'après AMpèine, à l'action électromagnétique exercée, sur ce même point, par un certain systène de courants constants et fermés qui circulent en partic dans l'espace $S$, en partie sur la surface $\sigma$ qui termine cet espace. Pour définir complètement ce système il faut connaitre les composantes $u, v, w$ de l'intensité spécifique (de volume) en tout point $(x, y, z)$ de l'espace $S$ et les composantes $\boldsymbol{u}, \boldsymbol{v}, \boldsymbol{v}$ de l'intensité spécifique (de superficie) en tout point $(x, y, z)$ de la surface $\sigma$. Or ceś six quantités sont données en fonction des composantes $\alpha, \beta, \gamma$ du moment magnétique par les formules très-simples

$$
\begin{array}{r}
u=\frac{\partial \beta}{\partial z}-\frac{\partial \gamma}{\partial y}, \quad v=\frac{\partial \gamma}{\partial x}-\frac{\partial \alpha}{\partial z}, \quad w=\frac{\partial u}{\partial y}-\frac{\partial \beta}{\partial x}, \\
(\mathrm{I})_{\mathbf{a}} \quad \boldsymbol{u}=\beta \frac{\partial z}{\partial n}-\gamma \frac{\partial y}{\partial n}, \quad \boldsymbol{v}=r \frac{\partial x}{\partial n}-\alpha \frac{\hat{\partial} z}{\partial n}, \quad \boldsymbol{v}=\alpha \frac{\partial y}{\partial n}-\beta \frac{\partial_{x}}{\partial n},
\end{array}
$$

où $n$ est la normale intérieure à la surface $\sigma$ (voir le Reprint de Tiromson, $\S 554$, ou la Note citéc ci-dessus).

Il est commode de transformer les trois dernières formules. On a d'abord

$$
\boldsymbol{u} \frac{\partial x}{\partial n}+\boldsymbol{v} \frac{\partial y}{\partial n}+\boldsymbol{w} \frac{\partial z}{\partial n}=0
$$


relation dont la nécessité découle de la définition même des quantités $\boldsymbol{u}, \boldsymbol{v}, \boldsymbol{v}$. Menons, ensuite, par le point $(x, y, z)$ de la surface deux éléments linéaires orthogonaux $d s$, $d \nu$ de cette surface, de telle manière que les trois directions $d s, d \nu, d n$ soient congruentes $i$ celles des axes positifs $\operatorname{des} x, y, z$. On tire alors des équations $(\mathrm{I})_{\mathrm{a}}$

$$
\boldsymbol{u} \frac{\partial x}{\partial \nu}+\boldsymbol{v} \frac{\partial y}{\partial \nu}+\boldsymbol{w} \frac{\partial z}{\partial \nu}+\alpha \frac{\partial x}{\partial s}+\beta \frac{\partial y}{\partial s}+\gamma \frac{\partial z}{\partial s}=0,
$$

et l'on reconnaît très-nisément que les quantités $\boldsymbol{u}, \boldsymbol{v}, \boldsymbol{v}$, assujetties à la condition (2), ne peuvent satisfaire à cette dernière équation, quel que soit le couple orthogonal $(d s, d \nu)$, qu'en prenant les valeurs $(\mathrm{I})_{\mathbf{a}}$. La dernière équation peut être encore simplifice. Soit $j$ l'intensité spécifique dont $\boldsymbol{u}, \boldsymbol{v}, \boldsymbol{v}$ sont les composantes suivant les axes, et soit $\boldsymbol{j}_{v}$ la composante de cette même intensité suivant une direction quelconque $\nu$ : on peut alors écrire

$$
j_{y} d s+\alpha d x+\beta d y+\gamma d z=0
$$

où $d x, d y, d z$ sont les composantes, suivant les axes, de l'ćlément linéaire $d s$, et où $\nu$ indique la direction de l'élément linéaire normal à $d s$, dans le sens précis qui a été indiqué ci-dessus.

Cela posé, nous considérerons une distribution de magnétisme trègparticulière. On sait déjà par Poisson que lorsque les composantes du moment magnétique satisfont aux conditions

$$
\begin{array}{cc}
\frac{\partial \alpha}{\partial x}+\frac{\partial \beta}{\partial y}+\frac{\partial \gamma}{\partial z}=0 & \text { en tout point de } S \\
\alpha \frac{\partial x}{\partial n}+\beta \frac{\partial y}{\partial n}+\gamma \frac{\partial z}{\partial n}=0 \quad \text { » }, ~ \gg \sigma
\end{array}
$$

la fonction potentièlle magnétique est partout nulle. Mais nous supposerons, en outre, qu'il existe une fonction $\varphi$ des points de $S$, telle que l'on ait

$$
\alpha=-\frac{1}{4 \pi} \frac{\partial \varphi}{\partial x}, \quad \beta=-\frac{1}{4 \pi} \frac{\partial \varphi}{\partial y}, \quad \gamma=-\frac{1}{4 \pi} \frac{\partial \varphi}{\partial z}
$$


et que l'on ait aussi, par conséquent,

$$
\begin{aligned}
& \triangle_{2} \varphi=0 \text { en tout point de } S, \\
& \frac{\partial \varphi}{\partial n}=0 \quad 》 \quad \gg \quad \sigma .
\end{aligned}
$$

Les égalités (3) exigent que les premières dérivées de $\varphi$ soient monodromes, continues et finies en tous les points de $S$. Si donc on veut pouvoir satisfaire en mème temps aux conditions $(3)_{a},(3)_{b}$, sans que $\varphi$ devienne = Const., il faut supposer que l'espace $s$ soit infini, ou, s'il est fini, qu'il ne soit pas simplement connexe; et, dans ce second cas, il faut que la fonction $\varphi$ ne soit pas monodrome dans cet espace. Cette fonction peut, en effet, être envisagée comme le potentiel des vitesses d'un liquide incompressible, mobile dans l'espace $S$. (Pour fixer les idées, il est bon d'avoir en vue le cas simple d'un espace $S$ fini et doublement connexe, c'est à dire d'une surface $\sigma$ tubulaire et rentrante.)

Les équations (I) donnent $u=v=w=0$, d'où il suit, qu'il n'y a pas de courants dans l'espace $S$. L'équation (2) a donne de son côté

$$
\dot{j}_{\nu} d s=\frac{d \varphi}{4 \pi}
$$

d'où il suit $\boldsymbol{j}_{\nu}=0$ pour $d \varphi=0$ : il n'y a donc de courants que sur la surface $\sigma$ et ces courants circulent le long des lignes d'intersection de cette surface avec les surfaces $\varphi=$ Const. Si la direction $\nu$ est celle du courant, on a, pour l'intensité vraie du courant qui circule dans la bande comprise entre les deux lignes contigïes $\varphi=$ Const., $\varphi+d \varphi=$ Const.,

$$
j d s=\frac{d \varphi}{4 \pi} .
$$

On peut dire aussi que l'intensité spécifique $j$ est donnée par la formule

$$
j=\frac{1}{4 \pi} \frac{\partial \varphi}{\partial N}
$$

où $N$ est la normale aux surfaces $\varphi=$ Const., de sorte que cette intensité i a la même expression, au signe près, que la densité d'une couche de niveau sur une de ces surfaces. D'me manicre on d'antre, on voit que 
la fonction $\varphi$ régit la marche aussi bien que l'intensité de tous les courants $\mathrm{du}$ système.

De l'équivalence de ce système avec le corps magnétique on conclut immédiatement que l'action des courants est nulle au dehors de l'espace $S$. Pour calculer cette action dans l'espace $S$ lui-mème, il suffit de sc rappeler qu'à l'intérieur d'un corps magnétique les composantes de la force, suivant la définition dite électromagnétique, sont données par les dérivées négatives de la fonction potentielle (qui est nulle dans notre cas) augmentées respectivement de $4 \pi \alpha, 4 \pi \beta, 4 \pi \gamma$. Cette force n'est d'ailleurs autre chose que celle due au système de courants équivalents: donc (3) les composantes cherchées sont

$$
-\frac{\partial \varphi}{\partial x}, \quad-\frac{\partial \varphi}{\partial y}, \quad-\frac{\partial \varphi}{\partial z} .
$$

On peut maintenant écarter tout à fait la considération du corps magnétique et envisager $\varphi$ comme fonction potentielle électromagnétique de courants existant en dehors de l'espace $S$. On a alors le théorème suivant, dans l'énoncé duquel on a rétabli la généralité des hypothèses essentielles:

Soit $\varphi$ la fonction potentielle électromagnétique d'un système $\Gamma$ de courants et soit $\sigma$ une surface, lieu géométrique de lignes de force extérieures relatives $a$ ces courants, et partageant l'espace infini en deux régions, $S$ et $S^{\prime \prime}$, dont la première ne renferme aucune partie de $\Gamma$. Si l'on fait parcourir cette surface par un système de courants (solenoïde neutre), dont l'intensité spécifique (de superficie) soit

$$
j=\frac{1}{4 \pi} \frac{\partial \varphi}{\partial N}
$$

$N$ étant la normale aux surfaces $\varphi=$ Const., l'action électromagnétique de ce système est nulle dans tout l'espace $S^{\prime}$ et est identique, dans tout l'espace $S$, a celle $d u$ système primitif $\Gamma$.

On peut ajouter que l'intensité totale du solénoïde est égale à celle du système $\Gamma$, lorsque les lignes de force dont $\sigma$ est le lieu géométrique entourent tous les courants de ce système.

L'analogie de ces énoncés avec ceux qui se rapportent aux couches de niveau ordinaires est évidente. Cette analogie parait encore plus 
complète si l'on tient compte d'une propriété importante établie dès 1870 par M. Boltzmand (Journal de BoRChaRD', t. 73) relativement au potentiel d'un solénoïde neutre sur lui-méme, potenticl qui est exprimé par

$$
-\frac{1}{8 \pi} \int \dot{\Delta_{1} \varphi} \varphi \cdot d S
$$

On peut encore généraliser le théorème précédent, de manière à comprendre le cas qui a été considéré par M. Bertrand par rapport aux distributions de masse ordinaires. On trouve alors que si le solénoïde orthogonal sépare deux partics, $I_{s}^{\prime}$ et $I_{S^{\prime}}$, du système $I$, son action en $S$ est égale $i$ celle de la partic $\Gamma_{S^{\prime}}$, tandis que son action en $S^{\prime \prime}$ est égale et contraire à celle de la partie $\Gamma_{s}$. (Cette différence de signes est naturellement subordonnée au choix que l'on a fait du sens des courants du solénoïle.)

Considérons un exemple très-simple. Supposons que le système I' se réduise à un seul courant rectiligne et indéfini, auquel cas les surfaces $\varphi=$ Const. sont des plans menés par cet axe. 'Toute surface de révolution autour de ce même axe peut jouer le rôle de surface $\sigma$, pourvu que la ligne móridienne ne coupe pas l'axe et soit une courbe fermée ou à branches infinies. Les courants de ce solénoïle étant distribués uniformément le long des lignes méridiennes et leur intensité totale étant égale à celle du courant axial, l'action du solénoïde dans l'espace annulaire est identique $i$ celle de ce courant. Par conséquent, s'il existe, dans cet espace annulaire, une masse de fer doux, l'induction magnétique exercée sur cette masse par le solénoïle est nécessairement la mème que si, le solénoïde étant supprimé, la masse était exposée à l'action du courant axial de méme intensité. D'apris cela, lorsque la masse de fer doux a elle-aussi la forme d'un anneau de révolution autour de l'axe (la section étant quelconque), sa surface devient un licu de lignes de force inductrices. Or chaque fois que la surface d'un corps magnétique isotrope est un lieu de lignes de force inductrices, la solution du problème d'induction est immédiatement donnée par la seule inspection de l'équation fondamentale de Poisson (la magnétisation induite rentre alors dans le type (3), (3) $(3)_{b}$, où $\varphi$ est proportionnelle à la fonction potentielle des forces inductrices extéricures). On est ramené ainsi, dans le cas très-particulier de l'anneau 
de révolution, au résultat que $M$. Kinchinofr a établi par le calcul direct (Gesammelte Abhandlungen, p. 226). ( $\left.{ }^{1}\right)$

Voyons maintenant l'application que l'on peut faire des propriétés précédemment établies aux surfaces de second ordre.

Désignons pas $u, v, w$ les trois racines réelles de l'équation en $\lambda$

$$
\frac{x^{2}}{a^{2}+\lambda}+\frac{y^{2}}{b^{2}+\lambda}+\frac{z^{2}}{c^{2}+\lambda}=\mathrm{I},
$$

en supposant

$$
\infty>u>-c^{2}>v>-b^{2}>w>-a^{2}
$$

et posons

$$
U=\int_{-c^{2}}^{u} \frac{d u}{\sqrt{F(u)}}, \quad V=\int_{-b^{2}}^{v} \frac{d v}{\sqrt{-F^{\prime}(v)}}, \quad W=\int_{-a^{2}}^{v} \frac{d w}{\sqrt{F^{2}(w)}},
$$

oü

$$
F(\lambda)=\left(a^{2}+\lambda\right)\left(b^{2}+\lambda\right)\left(c^{2}+\lambda\right) .
$$

Nous fixerons tout-à-l'heure le signe des radicaux sous les intégrales $U$, $V, W$ : en attendant, nous désignerons par $I, I, K$ les valeurs absolues de ces trois intégrales, lorsque les variables $u, v, w$ y atteignent leurs limites supérieures (respectives) $\infty,-c^{2},-b^{2}$.

On sait que l'élément linéaire de l'espace est donné, en $u, v$, w, par l'équation

$4 d s^{2}=(u-v)(u-w) d U^{2}+(u-v)(v-w) d V^{2}+(u-v)(v-w) d W^{2}$,

d'où l'on tire

$\triangle_{1} U=\frac{4}{(u-v)(u-w)}, \quad \triangle_{1} V=\frac{4}{(u-v)(v-w)}, \quad \triangle_{1} W=\frac{4}{(u-w)(v-v)}$.

(1) Il n'est pas inutile de remarquer qu'il y a deux cas généraux où le problème de l'induction magnétique admet une solution inmédiate. Le premier est celui où la surface qui termine le corps est de niveau par rapport aux forces inductrices: c'est le cas qui a servi à M. Thomson pour expliquer les résultats de Faraday au sujet de l'induction élcetrostatique. Le second est celui dont il est question ci-dessus. 
De ces dernières expressions on conclut, comme on sait, que les dérivées des fonctions $U, V, W$ par rapport à $x, y, z$ sont finies partout, excepté le long de l'ellipse focale, pour $U$, de l'hyperbole focale, pour $V$, et de ces deux lignes à la fois, pour $W$. Les dérivées secondes de ces trois fonctions satisfont aux équations

$$
\triangle_{2} U=0, \quad \triangle_{2} V=0, \quad \triangle_{2} W=0
$$

partout où elles sont finies.

D'après cela, si on considère un des hyperboloïdes à une nappe, que nous désignerons par $\sigma$ et qui sera défini par une valeur particulière $v_{0}$ de $v$, et si, pour distinguer entre elles les deux régions de l'espace qui sont séparées par cette surface, l'on indique par $S$ celle où se trouve le centre et par $S^{\prime}$ la région annulaire extérieure, on voit que les dérivées

$$
\frac{\partial U}{\partial x}, \quad \frac{\partial U}{\partial y}, \quad \frac{\partial U}{\partial z}
$$

sont finies en tout point de $S$ et que les dérivées

$$
\frac{\partial W}{\partial x}, \quad \frac{\partial W}{\partial y}, \quad \frac{\partial W}{\partial z}
$$

sont finies en tout point de $S^{\prime}$.

Pour que les dérivées de $U$ deviennent aussi continues en $S$, il suffit de changer le signe au radical $\sqrt{F(u)}$ chaque fois que le point $(x, y, z)$ traverse le plan $z=0$, où ce radical s'annule. De méme, pour que les dérivées de $W$ deviennent aussi continues en $S^{\prime}$, il suffit de changer le signe au radical $\sqrt{F(w)}$ chaque fois que le point $(x, y, z)$ traverse les deux plans $x=0, y=0$, où cc radical s'annule. Afin de satisfaire à ces deux conditions, nous conviendrons de donner toujours au radical $\sqrt{F(u)}$ le même signe que $z$ et au radical $\sqrt{F(w)}$ le signe contraire à celui du produit $x y$. Par ces conventions, lorsque le point $(x, y, z)$ ira de $z=-\infty$ à $z=+\infty$ par un chemin quelconque en $S$, la fonction monodrome $U$ passera avec continuité de la valeur $-H$ à la valeur $+H$; et lorsque le point $(x, y, z)$ décrira en $S^{\prime}$ un chemin fermé autour de l'hyperbolorde $\left(v_{0}\right)$, la fonction polidrome $W$ passera avec continuité de la valeur $W$, qu'elle possédait au point de départ, à la valeur $W+{ }_{4} K$ 
Sur les couches de niveau électromagnétiques.

qu'elle prendra au même point, après le tour complet (en sens positif). Par ces mêmes conventions, si l'on désigne par $s$ l'arc de l'une quelconque des courbes à branches infinies

$$
v=\text { Const. } \quad w=\text { Const. }
$$

qui existent dans l'espace $S$, et par $s^{\prime}$ l'arc de l'une quelconque des courbes fermées

$$
u=\text { Const. }, \quad v=\text { Const. }
$$

qui existent en $S^{\prime}$, on aura

$$
\frac{\partial U}{\partial s}=\frac{2}{\sqrt{(u-v)(u-v)}}, \quad \frac{\partial W}{\partial s^{\prime}}=\frac{2}{\sqrt{(u-w)(v-v)}}
$$

(radicaux toujours positifs), les arcs $s$ et $s^{\prime}$ croissant dans les sens indiqués ci-dessus: On voit donc que ces dérivées, ainsi 'que celles prises par rapport à $x, y, z$ (que l'on déduit immédiatement des précédentes), sont bien réellement monodromes, continues et finies dans les espaces (respectifs) $S$ et $S^{\prime}$, et qu'elles deviennent, à l'infini, infiniment petites du second ordre par rapport à l'inverse du rayon vecteur.

(On peut faire des considérations tout-à-fait analogues sur la fonction $\boldsymbol{V}$, qui est de l'espèce de $W$.)

Les fonctions $U$ et $W$ étant définies de la sorte, rien ne s'oppose désormais à ce que nous considérions ces fonctions comme des cas particuliers de celle que nous avions désignée par $\varphi$, c'est à dire que nous prenions $\varphi=U$, par rapport à l'espace $S$, et $\varphi=W$, par rapport à l'espace $S^{\prime}$. On a alors sur l'hyperbololde $\sigma$ deux systèmes de courants et on peut immédiatement énoncer les théorèmes suivants:

Si les lignes de courbure elliptiques de l'hyperboloïde d une nappe $\left(v_{0}\right)$ sont parcourues par des courants, avec l'intensité spécifique

$$
j=\frac{1}{4 \pi} \frac{\partial U}{\partial N}
$$

où $N$ est la normale aux ellipsoìdes, l'action électromagnétique de ce système de courants est nulle dans lespace $S^{\prime}$ et a la fonction potentielle $U$ (a une constante près) dans l'espace $S$. 
Si les lignes de courbure hyperboliques du même hyperboloüde sont parcourues par des courants, avec l'intensité spécifique

$$
\dot{j}=\frac{1}{4 \pi} \frac{\partial W}{\partial N}
$$

ò̀ $N$ est la normale aux hyperbolö̀les à deux nappes, l'action électromagnétique de ce second système de courants est nulle dans l'espace $S$ et a la fonction potentielle $W$ (à une constante près) dans l'espace $S^{\prime \prime}$.

On peut considérer $U$ comme la fonction potentielle de courants $I_{S^{\prime}}$ existant dans l'espace $S^{\prime}$ et $W$ comme la fonction potentielle de courants $I_{S}^{\prime}$ existant dans l'espace $S$. Ces courants peuvent prendre, évidemment, des dispositions très-variées. On peut, par exemple, concevoir $I_{S^{\prime}}^{\prime}$ comme étant formé de courants disposés suivant les lignes de courbure elliptiques d'un hyperbolö̈de à une nappe au paramètre $v>v_{0}$, ou mềne, à la limite, suivant les ellipses du plan $z=0$ homofocales et extérieures à l'ellipse focale: la loi de distribution de ces courants serait la même que pour ceux de l'hyperboloïde $\left(v_{0}\right)$; seulement il faudrait, dans le cas limite, en doubler l'intensité. On peut, par la mème ruison, concevoir $I_{s}$ comme étant formé de courants disposés suivant les lignes de courbure hyperboliques d'un hyperboloïde à une nappe au paramètre $v<v_{0}$, ou même, a la limite, suivant les hyperboles du plan $y=0$ homofocales et intérieures à l'hyperbole focale (avec les mêmes remarques touchant la loi de distribution).

$\mathrm{Si}$ on fait coexister, sur un mème hyperboloïde $\left(v_{0}\right)$, les deux systèmes de courants qui circulent le long des lignes de courbure, on obtient un nouveau système, dont les courants parcourent les lignes

$$
\varphi=U+W=\text { Const }
$$

ou les lignes

$$
\varphi=U-W=\text { Const, }
$$

suivant le sens des deux systèmes composants. Il est facile de voir que ces lignes ne sont autres que les génératrices rectilignes de l'hyperboloïde (ce qui n'est qu'une forme particulière du théorème d'addition). L'intensité 
du courant composé qui parcourt la bande comprise entre deux génératrices contigües est donnée par la formule générale

$$
\frac{d \varphi}{4 \pi}
$$

mais on peut aussi exprimer l'intensité spécifique au moyen de la troisième fonction $V$, que nous n'avons pas encore mise en oeuvre. En effet l'élément linéaire de l'hyperboloïde étant donné par l'équation

$$
\left.4 d s^{2}=(u-w)_{\{}\left(u-v_{0}\right) d U^{2}+\left(v_{0}-w\right) d W^{2}\right\},
$$

la condition d'orthogonalité de deux éléments $d s$, is du mème hyperboloïde est

$$
\left(u-v_{0}\right) d U \delta U+\left(v_{0}-w\right) d W i W=0 .
$$

Si le second élément appartient à l'une des génćratrices rectilignes, savoir si l'on a $\partial U \pm \partial W=0$, cette condition se réduit à

$$
\left(u-v_{0}\right) d U \mp\left(v_{0}-u\right) d V=0 .
$$

De cette équation et de $d U+d W=d \varphi$ on tire

$$
d U=\frac{v_{0}-w}{u-w} d \varphi, \quad d W=+\frac{u-v_{0}}{u-w} d \varphi,
$$

et, par suite, la distance normale $d s$ des deux génératrices $\varphi$ et $\varphi+d \varphi$, au point considéré, est donnée par

d'où l'on tire

$$
4 d s^{2}=\left(u-v_{0}\right)\left(v_{0}-w\right) d \varphi^{2}
$$

$$
\left(\frac{\partial \varphi}{\partial_{s}}\right)^{2}=\Delta_{1} V
$$

Liintensité spécifique des courants rectilignes peut donc s'exprimer, abstraction faite du signe (que l'on détermine aisément d'après les conventions), par

$$
j=\frac{1}{4 \pi} \frac{\partial V}{\partial N}
$$

où $N$ est la normale à l'hyperboloìde $\left(v_{0}\right)$. 
Ainsi l'on a cet autre théorème:

Le système des courants qui parcourent les génératrices rectilignes, d'une même famille, de l'hyperbolö̈le $\left(v_{0}\right)$, avec l'intensité spécifique

$$
j=\frac{1}{4 \pi} \frac{\partial V}{\partial N},
$$

où $N$ est la normale à cette même surface, a, dans l'espace $S$, la fonction potentielle $U$ (à une constante et au signe près) et, dans l'espace $S^{\prime}$, la fonction potentielle $W$ (à une constante et au signe près).

Le choix des signes de $U$ et de $W$ dépend du sens des courants rectilignes (nous omettons la discussion de ces signes, qui n'offre aucune difficulté).

On reconnait aisément que ce dernier théorème n'est qu'un cas particulier de celui que nous avions énoncé pour le cas où la surface $\sigma$ sépare le système $\Gamma$ en deux parties. Sous ce point de vue $\Gamma_{s}$ et $\Gamma_{s}$ pourraient être, par exemple, les deux couches focales (électromagnétiques).

D'après ce qui précède, les lignes de courbure et les lignes asymptotiques de l'hyperboloide à une nappe reçoivent une application électromagnétique très-simple. Dans cette application toutes les racines de l'équation cubique bien connue trouvent leur emploi, tandis qu'une seule de ces racines figure dans les applications qui se rattachent à la théorie du potentiel ordinaire. 